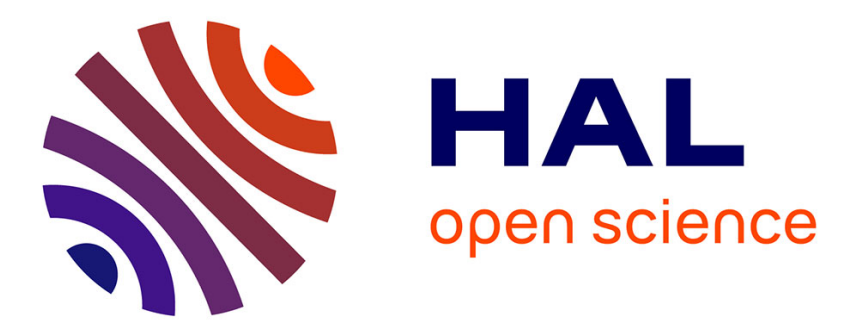

\title{
Pharmacokinetics and pharmacodynamics of stereoisomeric drugs with particular reference to bioequivalence determination
}

\author{
Peter Lees, Robert Hunter, Phil Reeves, Pierre-Louis Toutain
}

\section{To cite this version:}

Peter Lees, Robert Hunter, Phil Reeves, Pierre-Louis Toutain. Pharmacokinetics and pharmacodynamics of stereoisomeric drugs with particular reference to bioequivalence determination. Journal of Veterinary Pharmacology and Therapeutics, 2012, 35, pp.17-30. 10.1111/j.1365-2885.2012.01367.x . hal-01191249

\author{
HAL Id: hal-01191249 \\ https://hal.science/hal-01191249
}

Submitted on 1 Sep 2015

HAL is a multi-disciplinary open access archive for the deposit and dissemination of scientific research documents, whether they are published or not. The documents may come from teaching and research institutions in France or abroad, or from public or private research centers.
L'archive ouverte pluridisciplinaire HAL, est destinée au dépôt et à la diffusion de documents scientifiques de niveau recherche, publiés ou non, émanant des établissements d'enseignement et de recherche français ou étrangers, des laboratoires publics ou privés. 


\section{Pharmacokinetics and pharmacodynamics of stereoisomeric drugs with particular reference to bioequivalence determination}

\section{P. LEES*}

R. P. HUNTER ${ }^{\dagger}$

P. T. REEVES ${ }^{\ddagger} \&$

P. L. TOUTAIN $\$$

*Royal Veterinary College, Hatfield, UK; ${ }^{\dagger}$ Food Animal Therapeutics, Elanco Animal Health, Greenfield, IN, USA; ${ }^{\star}$ Australian Pesticides and Veterinary Medicines Authority, Canberra, ACT, Australia; ${ }^{\S}$ Ecole Nationale Veterinaire de Toulouse, 23 Chemin des Capelles 31076, Toulouse, France
Lees, P., Hunter, R. P., Reeves, P. T., Toutain, P. L. Pharmacokinetics and pharmacodynamics of stereoisomeric drugs with particular reference to bioequivalence determination. J. vet. Pharmacol. Therap. 35 (Suppl. 1), 17-29.

Drugs containing one or more chiral centres exist in stereoisomeric molecular forms. Most commonly, drugs containing a single asymmetric carbon atom exist in two enantiomeric forms, designated as eutomer (the more potent) and distomer (the less potent). As well as differences in potency and other pharmacodynamic properties, most members of enantiomeric pairs commonly differ also in their pharmacokinetic profiles. This article reviews factors underlying differences in pharmacological properties of enantiomers. The relevance of such differences for studies designed to evaluate the bioequivalence of products containing chiral drugs is also reviewed.

(Paper received 15 December 2011; accepted for publication 16 December 2011)

Professor Peter Lees, Royal Veterinary College, Hawkshead Lane, Hatfield, Herts AL9 7TA, UK. E-mail: plees@rvc.ac.uk

\section{INTRODUCTION}

This paper reviews the nature of chirality and its impact on drug pharmacokinetics and in particular its relevance to veterinary bioequivalence. The body is a chiral environment, containing, for example, D-carbohydrates and proteins based on L-amino acids. Most drugs (as well as hormones, autacoids and neurotransmitters) produce responses through interaction with target sites (enzymes and receptors), which are protein in structure. Moreover, for most drug actions, a 3-point fit at the target site is required for optimal activity. Therefore, it is generally the case that stereoisomers of drug molecules containing one or more chiral centres have differing potencies for pharmacological and/or toxicological effects. Chirality also, for many drugs, has a role in determining the rate and extent of the pharmacokinetic processes: absorption, distribution, metabolism and excretion.

Bioequivalence for two or more formulated products or for two or more routes of administration of a single product is, within predefined statistical limits, determined by the rate and extent of absorption of the active constituents. Therefore, it may be appropriate, in some instances, to apply bioequivalence criteria to each of the separate enantiomers or at least to the eutomer of an enantiomeric pair, in products containing a chiral drug marketed as the racemate. In other cases, determination of bioequivalence for 'total drug' may be acceptable. Moreover, there is no international harmonization on this issue. The question of using chiral or nonchiral analyses is topical and controversial and therefore appropriate for review at this time. For bioequivalence determination, this article (i) reviews general principles on which to base decisions on analytical methodology (chiral or nonchiral [achiral] assay); (ii) presents the often conflicting views; (iii) considers selected case histories from a large literature as the basis for formulating future guidance on when to adopt enantiomeric analytical methods; and (iv) offers comment to organizations concerned with developing international harmonization guidelines for veterinary drug products.

\section{NATURE AND NOMENCLATURE OF MOLECULAR ISOMERISM}

Isomers are chemical compounds with the same molecular formula. Categories of isomers and definitions are presented in Fig. 1.

Phosphorus, sulphur and nitrogen atoms, as well as carbon, can form chiral molecules. However, given that the molecules of both living matter and therapeutic drugs are based very largely on carbon, the most important example of asymmetry in biochemistry and pharmacology is the orientation of four different groups attached to a tetravalent carbon atom. An example is the nonsteroidal anti-inflammatory drug (NSAID) carprofen (Fig. 2). Occasionally, biologically active molecules have more than one centre of asymmetry and then several stereoisomers $(n)$ exist, where $n=2^{x}$ and $x$ is the number of asymmetric centres. For example, alkaloids from the bark of the Cinchona tree include quinine and quinidine. However, the number of asymmetric carbon atoms is four, at C3, C4, C8 and C9, so that 16 optically active isomers are possible, although only four - quinine, epiquinine, quinidine and epiquinidine occur in nature. The former two compounds and the latter two 


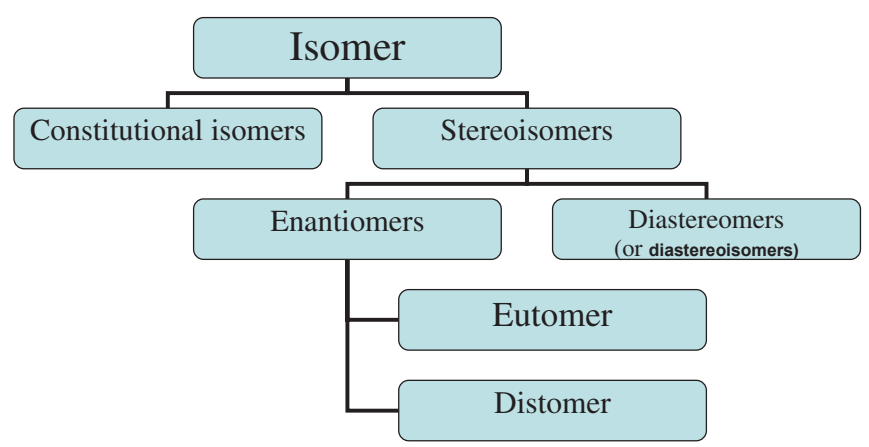

Fig. 1. Definition and classification of isomers.

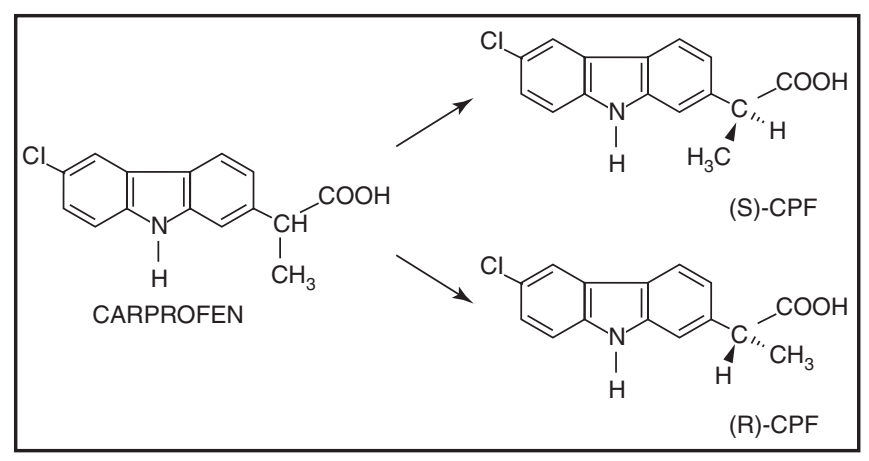

Fig. 2. Structures of carprofen enantiomers.

compounds each (separately) comprise an enantiomeric pair, whereas quinine and quinidine are diastereomers.

Two principal nomenclature systems have been used to describe the absolute configuration of asymmetric molecules. The Fisher convention uses two reference compounds, the natural levorotatory enantiomer of the amino acid serine (designated L-serine) and the natural dextrorotatory enantiomer of glyceraldehyde (designated D-glyceraldehyde). An alternative system was introduced by Cahn et al. (1956). It is based on rules that assign an order of decreasing priority $(\mathrm{a}-\mathrm{d})$ to each of the groups attached to the asymmetric carbon, decreasing atomic number being the simplest consideration. The molecule is then viewed with the lowest priority group orientated away from the viewer. If the direction of rotation from the highest to the lowest priority group is to the left, the enantiomer is designated $\mathrm{S}$ (sinister), and if the direction of rotation is to the right, the molecule is designated $\mathrm{R}$ (rectus). The existence of these two systems of nomenclature has led to difficulties, for example rendering recognition and description of the biological properties of enantiomers sometimes difficult. In addition, a third system of signs has been proposed by Simonyi et al. (1989).

\section{PROS AND CONS OF USING RACEMATES IN THERAPEUTICS}

Ariens (1986) was a particularly articulate and vociferous opponent of the therapeutic use of mixtures (usually racemic) of enantiomeric drug pairs. He referred to stereochemistry as a potential 'basis for highly sophisticated nonsense in pharmacokinetics and clinical pharmacology'. He further describes the administration of racemic mixtures as 'polypharmacy' with no therapeutic rationale. Moreover, he clearly and correctly classified racemic mixtures of drugs with a single chiral centre as mixtures of two drugs, only one of which is (in many cases) likely to contribute to the pharmacological property providing the basis for therapeutic use. There is the danger that the distomer contributes little or nothing to therapeutic response, whilst possibly possessing toxic side effects as discussed above.

Even when there is lack of both pharmacological and toxicological activity of the distomer, there remain other concerns on the use of racemates in products formulated for therapeutic use. As Ariens (1986) pointed out, an inactive enantiomer merely provides 'therapeutic ballast'. An impurity at the level $50 \%$ of the active constituent would never be tolerated by regulatory authorities for any other constituent, which is neither an active, nor excipient nor solvent. Moreover, even a biologically inactive enantiomer imposes an unnecessary load on the body's pathways, generally renal and/or hepatic, for drug elimination.

For many years, the availability of racemic mixture drug products, in preference to single enantiomers, was justified by lower production costs and/or lack of availability of chirally specific drug synthesis methods (Nerurkar et al., 1992). Unlike living systems, which generally synthesize chiral compounds in enantiomerically pure forms, laboratory synthesis of drugs containing a centre of asymmetry usually yields the optically inactive racemate. Hence, many drug products were developed as racemates; the chemical synthesis of pure enantiomers is almost invariably more expensive, especially on a commercial scale. The alternative route to preparation of a single enantiomer is to utilize nonchiral synthesis with subsequent separation. In the early days of racemic drug product development, analytical methods for separating and quantifying single enantiomers were commonly not available. These considerations now carry much less weight but, even so, it can be argued that the development of single enantiomer products to replace existing racemate-based products can often not be justified economically, as extensive and expensive new studies on efficacy and new laboratory animal and target species toxicity data, as well as human food safety data for food-producing species, may be required by regulatory authorities. It is further argued that racemate-based products may have good records in clinical use in terms of efficacy and pharmacovigilance: 'If it is not broken why fix it?'

\section{THE BODY AS A CHIRAL ENVIRONMENT}

\section{Physiological and biochemical consequences}

Early descriptions of stereoisomerism recognized the fact that living systems discriminate between stereoisomers. Indeed, Van't Hoff (1898) described differentiation by biological systems as a criterion for establishing that stereoisomers are distinct 
compounds. The pioneering studies of Cushny, Dale, Brown and others in the first decades of the 20th century led to discovery of the fact that the potency for action on the autonomic nervous system of each stereoisomer of adrenaline, nicotine and hyoscine differed markedly from the other. It was later recognized that many hormones and neurotransmitters (e.g. L-noradrenaline, Ladrenaline, L-dopamine, L-thyroxine and D-aldosterone) are synthesized in the body in enantiomerically pure forms and their potencies are considerably greater than those of their enantiomeric pairs. Chirality is important in biochemical processes when a close steric fit of a hormone, transmitter or autacoid into the active binding site in a protein (enzyme or receptor) involves either the chiral centre directly or a part of the active molecule close to the chiral centre. Affinity for attachment then differs for a pair of enantiomers, that is, chiral discrimination will occur.

\section{Pharmacological consequences}

\section{Introduction.}

Several reviews (Caldwell et al., 1988; Campbell, 1990; Delatour et al., 1994; Nation \& Sanson, 1994; Landoni et al., 1997; Brocks, 2006) have described the basis for pharmacological differences between the enantiomers of chiral drug molecules. The reader is referred to these reviews for fuller descriptions of pharmacological differences and also for accounts of analytical methods used to separate and quantify the isomers of an enantiomeric pair. A brief and selective account only is provided here. Stereospecificity may occur in pharmacodynamic, toxicodynamic and pharmacokinetic (absorption, distribution, metabolism and excretion) processes, in particular those that utilize a carrier protein, a receptor or an enzyme. It has been estimated that approximately $20-25 \%$ of drugs used clinically are optically active. They include many plant alkaloids and glycosides used for centuries in medicine. For example, the dried exudate of the oriental poppy Papaver somniferum yields opium, the main constituent of which is (-) morphine, and the common foxglove Digitalis purpurea is the source of $(+)$ digoxin.

\section{Pharmacodynamics.}

Three properties define the pharmacodynamic profile of a drug: (i) efficacy $\left(E_{\max }\right)$, the maximum response attainable; (ii) potency (usually expressed as $\mathrm{EC}_{50}$, although other values, e.g. $\mathrm{EC}_{80}$, $\mathrm{EC}_{90}$, may be more useful), the drug concentration producing $50 \%, 80 \%$ and $90 \%$ of $E_{\text {max }}$; and (iii) $N$, the slope/steepness of the concentration-effect relationship. For drugs with inhibitory actions, corresponding terms are $I_{\max }, \mathrm{IC}_{50}$, etc. (Toutain \& Lees, 2004).

There is an extensive literature on potency differences of drug molecule enantiomers. Quantitatively, each member of an enantiomeric pair commonly produces the same biological effect but with differing potency, in which case the relationship is that of eutomer and distomer. The eudismic or potency ratio is defined as the ratio (of the doses for in vivo studies or the concentrations for in vitro studies) producing effects of the same intensity (Ariens, 1986). For example, the sympathomimetic drug dobu- tamine exists as $(+)$ and $(-)$ enantiomers. The former is a $\beta$ adrenoceptor agonist and the latter is an agonist for $\alpha$ adrenoceptors. Use of the racemate has been preferred clinically, as both enantiomers are positive inotropes, but opposing vascular effects minimize the pressor effect owing to the vasoconstrictor action of (-) dobutamine. Similarly, both (+) and (-) enantiomers of bupivacaine are local anaesthetics but only the (-) enantiomer is vasoconstrictor, an action that is useful in increasing the duration of action at the administration site.

In an early review, Evans (1992) described the potency of several 2-arylpropionic acid agents of the nonsteroidal antiinflammatory drug (NSAID) class. These drugs include carprofen, ketoprofen and vedaprofen. Each has a single chiral centre and the enantiomers are designated $S(+)$ and $R(-)$. The principal mode of action at the molecular level is the inhibition of cyclooxygenase (COX), a key enzyme in the arachidonic acid cascade that generates pro-inflammatory mediators, such as prostaglandin $(\mathrm{PG}) \mathrm{E}_{2}$ and the pro-aggregatory autacoid thromboxane $A_{2}$. Cyclooxygenase exists in two isoforms: COX-1, concerned with blood clotting and gastroprotection, and COX-2, which is generated at sites of inflammation and which causes hyperalgesia and local oedema.

Potential differences in both potency and potency ratios for 2arylpropionate enantiomers, as inhibitors of COX-1 and COX-2 isoforms, are illustrated by whole blood in vitro assays for $\mathrm{S}(+)$ and $\mathrm{R}(-)$ carprofen (Table 1 ). It will be seen that (i) $\mathrm{S}(+)-$ carprofen is the more potent enantiomer for the inhibition of both COX isoforms in both horses and dogs; (ii) there are differences in potency for $\mathrm{S}(+)$ carprofen between horses and dogs for both isoforms; (iii) there are no differences in potency for $\mathrm{R}(-)$ carprofen between horses and dogs; (iv) the COX-1:COX-2 potency ratio for $\mathrm{R}(-)$ carprofen is low in both species, and it is therefore classified as nonselective; and (v) $\mathrm{S}(+)$-carprofen is classified as a selective inhibitor of COX-2 in dogs and a nonselective inhibitor in horses.

If some relationship (however complex) between COX inhibition and both therapeutic response and target species safety is assumed, two conclusions may be drawn from the data in Table 1. First, the apparent species differences in potency $\left(\mathrm{IC}_{50}\right)$ of COX-2 inhibition will imply a requirement for differing plasma and biophase concentrations for a given magnitude of clinical response. Second, the differing $\mathrm{IC}_{50} \mathrm{COX}-1: \mathrm{IC}_{50} \mathrm{COX}-2$ potency

Table 1. $\mathrm{IC}_{50}$ values for COX-1 and COX-2 inhibition and $\mathrm{IC}_{50}$ ratios

\begin{tabular}{lccc}
\hline Enantiomer & $\begin{array}{c}\text { COX-1 } \\
\mathrm{IC}_{50}(\mu \mathrm{M})\end{array}$ & $\begin{array}{c}\text { COX-2 } \\
\mathrm{IC}_{50}(\mu \mathrm{M})\end{array}$ & $\begin{array}{c}\text { COX-1: } \\
\text { COX-2 } \mathrm{IC}_{50} \text { ratio }\end{array}$ \\
\hline Dogs & & & \\
S $(+)$ carprofen & 176 & 7 & $25: 1$ \\
R(-) carprofen & 380 & 161 & $2.4: 1$ \\
Horses & & & \\
S $(+)$ carprofen & 25 & 14 & $1.7: 1$ \\
$\mathrm{R}(-)$ carprofen & 373 & 137 & $2.7: 1$ \\
\hline
\end{tabular}

COX-1, COX-2 ratios for $\mathrm{S}(+)$ and $\mathrm{R}(-)$ carprofen in the dogs and horses (whole blood assays). Data from Lees et al. (2004). 
ratios might imply species differences in safety for some classical side effects of NSAIDs, for example gastrointestinal toxicity and inhibition of haemostatic pathways. $S(+): R(-)$ potency ratios derived from quantifying drug action at the molecular level may differ from potency ratios in which the end-point comprises an in vivo pharmacological or toxicological measure, for example analgesia. There are several possible reasons for this, including drug response in vivo arising through molecular mechanisms other than or additional to COX inhibition. However, also involved in in vivo/in vitro differences will be the differing experimental circumstance of investigating drug action at a usually fixed concentration in vitro compared to the varying plasma and biophase concentrations occurring with time in vivo, that is, the additional consideration of differing pharmacokinetic profiles in the whole animal (vide infra). Despite these important differences between in vitro and in vivo studies and also between each in vivo response, in almost all instances the $\mathrm{S}(+)$ enantiomer of 2-arylpropionates is the eutomer with little activity residing in the $\mathrm{R}(-)$ enantiomer. However, further complicating the pharmacodynamic picture is the proposal by a minority of authors that $\mathrm{R}(-)$ enantiomers may contribute to therapeutic (analgesic, antipyretic, anti-inflammatory) responses. Lötsch et al. (1995) described an analgesic action of $\mathrm{R}(-)$ flurbiprofen, which did not correlate with COX inhibition. Therefore, the role of $\mathrm{R}(-)$ enantiomers, if any, in contributing to 2-arylpropionate therapeutic and side effects is not definitively understood.

\section{Toxicodynamics.}

In addition to quantitative differences in potency of enantiomeric drug pairs, qualitative differences can occur, so that each may exert differing actions, and this may have toxicological implications. For example, (-) amphetamine is a peripheral sympathomimetic, whereas (+) amphetamine is a CNS stimulant. Similarly, several barbiturates, a drug class that has been in veterinary use for more than 70 years (but now declining), exist as $\mathrm{S}(+)$ and $\mathrm{R}(-)$ enantiomers. The former cause CNS excitation, whilst the latter are CNS depressant. An extreme example is provided by 1-methyl-5-phenyl-propylbarbituric acid, the $\mathrm{R}(-)$ enantiomer of which is depressant and devoid of convulsant activity, whilst the $\mathrm{S}(+)$ enantiomer possesses no anaesthetic activity but causes dose-related tonic and clonic convulsions (Ticku et al., 1985). As agents such as pentobarbitone are used as racemates, there is a clear basis for improving the quality of anaesthesia by use of single enantiomers.

An important historical example of drug toxicity in therapeutic use is the birth defects in humans caused by racthalidomide administration to women in the early stages of pregnancy in the 1960s. It has been suggested that these might have been avoided by the use of a single enantiomer product rather than the racemate. This conclusion is based on the fact that the (R)-enantiomer is sedative whilst (S)-thalidomide is teratogenic. However, thalidomide is optically unstable in solution. The pure isomers of thalidomide racemize as a consequence of opening of the phthalimide ring, with half-lives for this process of 4-5 min in buffer at $\mathrm{pH} 7.4$ and of $<10 \mathrm{~min}$ in blood (Testa et al., 1993).
A veterinary example is tetramisole, an anthelmintic formerly used in pig and sheep medicine. It is a mixture of two enantiomers, both of which are active at nicotinic cholinergic sites (a basis for side effects in the animal host through stimulation of autonomic ganglia and also at the motor end plate of skeletal muscle) but only one of which, levamisole, is anthelmintically effective. As the racemic mixture is no longer marketed, the possibility of unwanted side effects from its use, particularly when toxicity may be difficult to identify and quantify, does not pose a risk.

\section{Pharmacokinetics.}

The impact of stereoselectivity on pharmacokinetics has been reviewed by Brocks (2006). Pharmacokinetic differences between enantiomeric pairs are of special relevance to bioequivalence considerations, when drugs are administered as racemates, as the definition of bioequivalence is similarity of rate and extent of absorption of the active constituents, as established by predefined, statistical criteria. Physicochemical properties of enantiomeric pairs are usually identical (or very similar). This includes lipid solubility, the property that predominantly governs passage of drugs across cell membranes by passive diffusion. Therefore, absorption from administration sites can be expected usually to be identical for an enantiomeric pair. Stereospecificity in drug absorption from the gastrointestinal tract is most likely when it involves transport by a carrier molecule. This is common for natural amino acids and sugars but less common for drugs. Some $\beta$-lactam antibiotics are transported by an intestinal dipeptide transport mechanism (Tamai et al., 1988). Stereoselectivity in gastrointestinal tract absorption has also been shown for the L-enantiomers of dopa and methotrexate, which are actively absorbed (Nerurkar et al., 1992). Wang et al. (2010) demonstrated stereoselective transport of propranolol enantiomers across Caco-2 cell monolayers. However, passive processes, such as diffusion of lipid-soluble molecules across gastrointestinal tract membranes, do not involve macromolecular interactions, and stereochemistry is then usually of limited or no consequence. An indirect cause of enantioselective absorption can arise, however, if enantiomers are vasoactive and differ in vasodilator or vasoconstrictor actions at sites of absorption. A more common basis for differing rates of uptake of members of an enantiomeric pair into the systemic circulation is differing rates of first-pass metabolic effect after oral dosing. Biotransformation may occur for some drugs in enterocytes and the lung, as well as the liver, before they reach the systemic circulation.

Tucker and Lennard (1990) and Nerurkar et al. (1992) reviewed stereospecificity in relation to drug binding to plasma and tissue proteins. For acidic chiral drugs, $S(+): R(-)$ plasma protein binding ratios were of the order of 1.3-1.7:1, whilst for most weak bases $S(+): R(-)$ ratios were in the range 0.6-1.7:1. The binding ratios, in most instances, reflect a diastereoisomeric association with albumin, the main binding protein for most acidic drugs, and $\alpha_{1}$-acid glycoprotein, which binds mainly basic drugs. Concentrations of $\alpha_{1}$-acid glycoprotein increase in inflammatory conditions, and this may affect the pharmacokinetics of basic drugs, whether chiral or nonchiral. Stereoselectivity in 
drug distribution may be a consequence of differences in plasma protein binding. This was proposed to explain the stereoselective accumulation of $\mathrm{S}(+)$-ibuprofen in human synovial fluid (Day et al., 1988). The transfer of drugs from the systemic circulation to the gut lumen by either biliary excretion (enterohepatic recycling; vide infra) or secretion by the cells of the gastrointestinal mucosa/submucosa (Ilett et al., 1990) may also demonstrate stereoselectivity.

Tissue binding is another cause of enantiomeric differences in drug distribution. For example, R(-) enantiomers of some 2arylpropionates, for example ibuprofen, are taken up selectively by fat, and a much greater uptake of both antipodes occurred when racemic or $\mathrm{R}(-)$ rather than $\mathrm{S}(+)$ forms was administered (Williams et al., 1986). This is not due simply to distribution, as metabolism is involved; selective formation of the coenzyme A-thioester of the Renantiomer and inversion to the S-thioester occurs in the liver and this is followed by incorporation of both esters as unnatural triglycerides. The toxicological consequences of this are not known. Stereoselectivity in volume of distribution has been demonstrated, in humans, for verapamil and warfarin (Nerurkar et al., 1992).

There are intrinsically differing rates of biotransformation of most 2-arylpropionates, in several species of veterinary interest. Thus, for many 2-arylpropionates, clearance differs between the two enantiomers; one basis for this is a chiral inversion mechanism of $\mathrm{R}(-)$ to $\mathrm{S}(+)$ enantiomers. This occurs for ketoprofen and vedaprofen. For carprofen, which does not undergo chiral inversion, clearance of the $\mathrm{S}(+)$ enantiomer in most species is more rapid than that of $\mathrm{R}(-)$ carprofen, so that $\mathrm{R}(-): \mathrm{S}(+)$ ratios for plasma area under curve (AUC) exceed 1:1. Moreover, these ratios are species dependent, and values of 4.6:1 (horses), 2.2:1 (cats), 1.5:1 (dogs) and 1.4:1 (calves) have been reported (Lees et al., 2004). In horses, the predominance of $\mathrm{R}(-)$ carprofen was shown to be due to a greater rate and degree of glucuronidation of the $\mathrm{S}(+)$ enantiomer (Soraci et al., 1995).

Ketoprofen provides an interesting example of supplementation of intrinsic clearance differences between species by chiral inversion, which is unilateral, $\mathrm{R}(-)$ to $\mathrm{S}(+)$. Calculated inversion rates were reported by Lees et al. (2004) as 49\% in horses, 32\% in calves, $22 \%$ in cats and $6 \%$ in sheep. For other agents of the 2 arylpropionate NSAID class, inversion, when it occurs, is usually solely from $\mathrm{R}(-)$ to $\mathrm{S}(+)$ but inversion from $\mathrm{S}(+)$ to $\mathrm{R}(-)$ has been described (Tanaka et al., 1992) and can account for in vivo racemization. This was shown to occur in dogs for the analgesic drug, oxindanac, irrespective of which enantiomer was administered (King et al., 1994). The major organ responsible for chiral inversion is the liver, but it may also occur in other tissues (kidney, fat, muscle and lung) (Berry \& Jamali, 1991).

From all the aforementioned data, the importance of quantifying each enantiomer separately in pharmacokinetic studies will be clear. As pointed out by Tucker and Lennard (1990), if both enantiomers of a racemic mixture decline monoexponentially, but at different rates, the pharmacokinetics of the combination appears artefactually to be biexponential. Two one-compartment models may be converted into a wholly spurious two-compartment model, if a nonstereospecific analytical method is used. The meaning and value of the calculated parameters will be, at best, questionable and, at worst, may lead to wrong conclusions. Nevertheless, achiral assays can, in some cases, be used in bioequivalence studies (vide infra).

Another drug group that warrants particular consideration, in view of their widespread use in veterinary medicine and availability in both pioneer and generic formulations, is the benzimidazole anthelmintics, fenbendazole, oxfendazole and albendazole. The sulphide benzimidazoles, albendazole and fenbendazole possess anthelmintic activity. They are prochiral molecules, which are converted in vivo to the chiral sulphoxides, albendazole oxide and fenbendazole oxide (oxfendazole), respectively. Most activity is attributable to the sulphoxides. The sulphoxide metabolites, are converted in vivo to inactive sulphones. These reactions were outlined in detail in the classical studies of Delatour et al. (1990a,b, $1991 a, b)$. They reported changes in plasma enantiomer concentration ratios with time. Thus, in sheep, after oral administration of fenbendazole, the ratio of oxfendazole enantiomers changed from 1.8:1 to $6.7: 1$ between 9 and $120 \mathrm{~h}$. Corresponding values for albendazole sulphoxide were $3.3: 1$ at $3 \mathrm{~h}$ and $22.4: 1$ at $36 \mathrm{~h}$. Plasma AUC ratios for the sulphoxide metabolites, following administration of the parent sulphides, were 26:74 for oxfendazole and 14:86 for albendazole sulphoxide. The authors postulated that these differences were either because of substrate enantioselectivity of cytochrome-dependent sulphonation or of differences in plasma protein binding leading to different renal clearance rates (Delatour et al., 1990b). Delatour et al. (1991a) also reported enantiomer $(+)$ :(-) ratios for albendazole sulphoxide, after administration of albendazole in humans, dogs and rats. Ratios changed linearly with time to reach values of 13.1:1 (humans), 9.3:1 (dogs) and 0.6:1 (rats). Overall, the (+) enantiomer represented, respectively, $80 \%$, $70 \%$ and $41 \%$ of total plasma AUC. The AUC of (+) albendazole sulphoxide, as a percentage of total, in another study in ruminants, was $80 \%, 86 \%$ and $91 \%$, in goats, sheep and cattle, respectively (Delatour et al., 1991b).

In summary, several authors have reviewed the influence of chirality on drug biotransformation. The stereospecific pharmacokinetic and pharmacodynamic profiles of several drugs of veterinary interest (ketamine, cloprostenol and the inhalational anaesthetics, desflurane, enflurane, halothane and isoflurane) were reviewed by Landoni et al. (1997). Caldwell et al. (1988) defined five categories of biotransformation: prochiral $\rightarrow$ chiral, chiral $\rightarrow$ chiral, chiral $\rightarrow$ diastereomer, chiral $\rightarrow$ achiral and chiral inversion. In addition to general differences in absorption, distribution, metabolism and excretion for chiral molecules, Nerurkar et al. (1992) discussed three other factors, which may complicate bioequivalence studies, namely nonlinear pharmacokinetics, genetic polymorphism and chiral inversion (vide infra).

\section{STEREOISOMERISM AND BIOEQUIVALENCE}

\section{Decision trees on choice of analytical method}

Until the late 1980s, there was limited discussion concerning the impact of stereoisomerism on bioequivalence studies. This changed in the 1990s, with the increased (and still increasing) 
availability of analytical methods, providing both complete separation and quantification of pure enantiomers in biological fluids such as plasma (Jamali, 1992; Nitchuk, 1992; Wechter, 1992; Marzo, 1993). A choice could then be made between the use of nonchiral (the 'total drug' concept) or chiral methods of analysis. There are available: (i) chiral derivatization reagents to convert enantiomeric substrates into diastereoisomeric complexes for separation on nonchiral columns and (ii) chiral stationary phase columns, which allow direct separation without diastereoisomerization (Landoni et al., 1997). Other means of resolution have been discussed by Srinivas (2004). The central question then and now is, 'Should the standard confidence interval criteria used to declare average bioequivalence be based on total drug, one enantiomer alone or all (usually both) enantiomers separately?' The question is simple but the answers may be complex and it may be necessary to make case-by-case decisions.

Bioequivalence is universally recognized as a means of establishing, within predefined limits, sufficient similarity in rate and extent of absorption of active constituents from two products (pioneer and generic) or two administration routes (e.g. intramuscular and subcutaneous). It is then accepted that, when used as alternatives, the products/routes can be expected to provide sufficiently similar efficacy and safety to guarantee therapeutic equivalence. The differences between and procedures required to establish average, individual and population bioequivalences are not considered here (see reviews by Barrett et al., 2000 and Gould, 2000), as all regulatory authorities require that data satisfy only the criteria for average bioequivalence. Also not reviewed are the arguments for and against the use of chiral/nonchiral methods for drug residue studies in food- producing species, except to note that residue depletion profiles depend on rate of depletion in the terminal elimination phase. For bioequivalence studies, the data requirements relate solely to rate and extent of drug absorption. Absorption is the crucial pharmacokinetic process. There are, however, common circumstances in veterinary medicine when flip-flop pharmacokinetics occurs and this can apply to stereoisomeric drugs. Moreover, after oral dosing, stereospecific absorption may apply to active as well as passive transport and stereoselective binding to plasma proteins can occur (vide supra).

Karim (1996) proposed an algorithm/decision tree approach, which was modified by Midha et al. (1998) (Fig. 3). Despite many subsequent deliberations and publications, there remains no international harmonization on this subject, nor is there universal acceptance of the algorithm outlined in Fig. 3. The algorithm allocates stereoisomeric drugs into three main categories (I, II, III) and then into subcategories (IIa, IIb, IIIa, IIIb) as defined in Fig. 3. In summary, the algorithm proposes that stereoselective methods are required in only two circumstances: first, when first-pass metabolic effect for the eutomer is high, resulting in changes in $S: R$ plasma concentration ratios, then both total and eutomer concentrations should be measured; second, when there is low first-pass metabolic effect for the eutomer and when also a specific S:R plasma concentration ratio is important for optimal therapeutic efficacy and/or safety. In all other cases, Midha et al. (1998) proposed that nonstereoselective analytical methods should suffice.

Mehvar and Jamali (1997) proposed an alternative decision tree (Fig. 4). They commented as follows:

In this paper, we have reviewed some theoretical and experimental evidence, indicating that the bioequivalence of some chiral drugs may

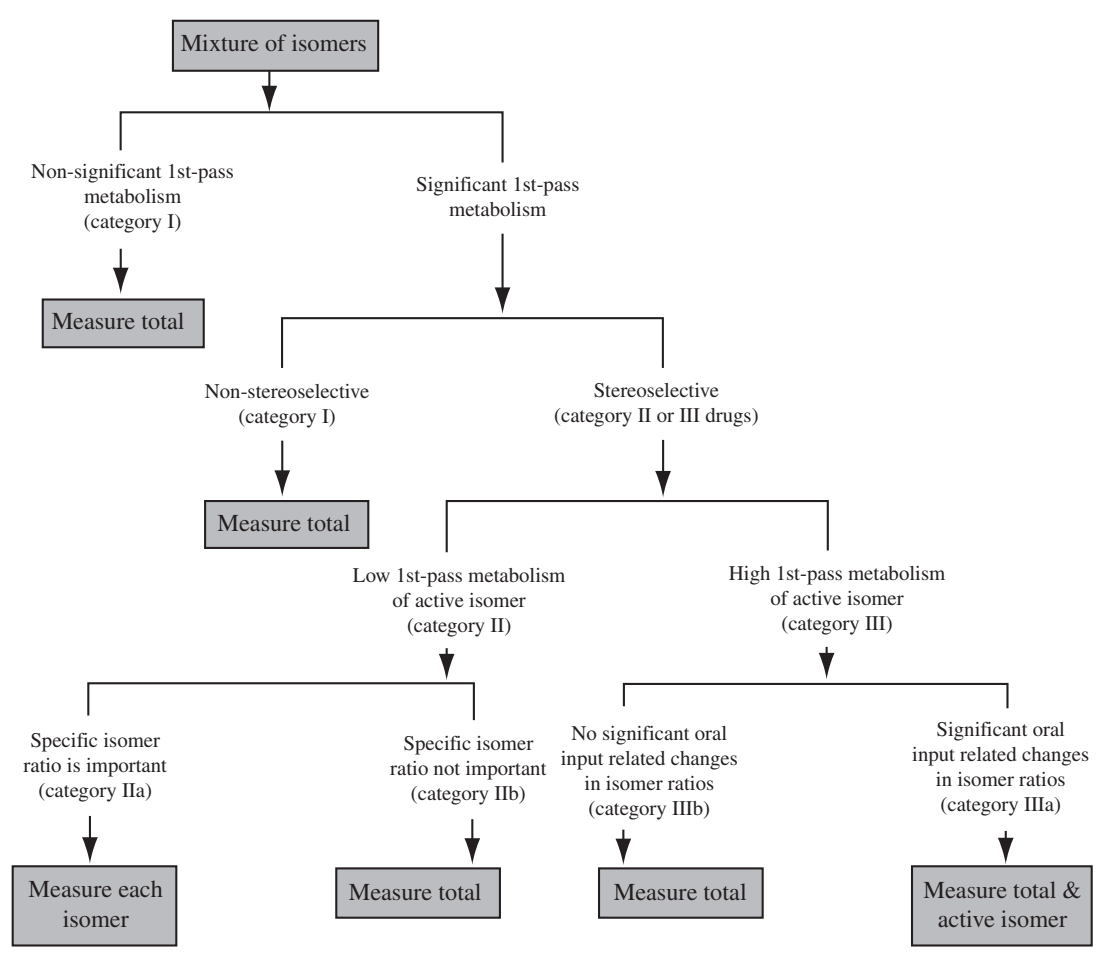

Fig. 3. Algorithm adapted from Midha et al. (1998). 
Fig. 4. Algorithm adapted from Mehvar and Jamali (1997).

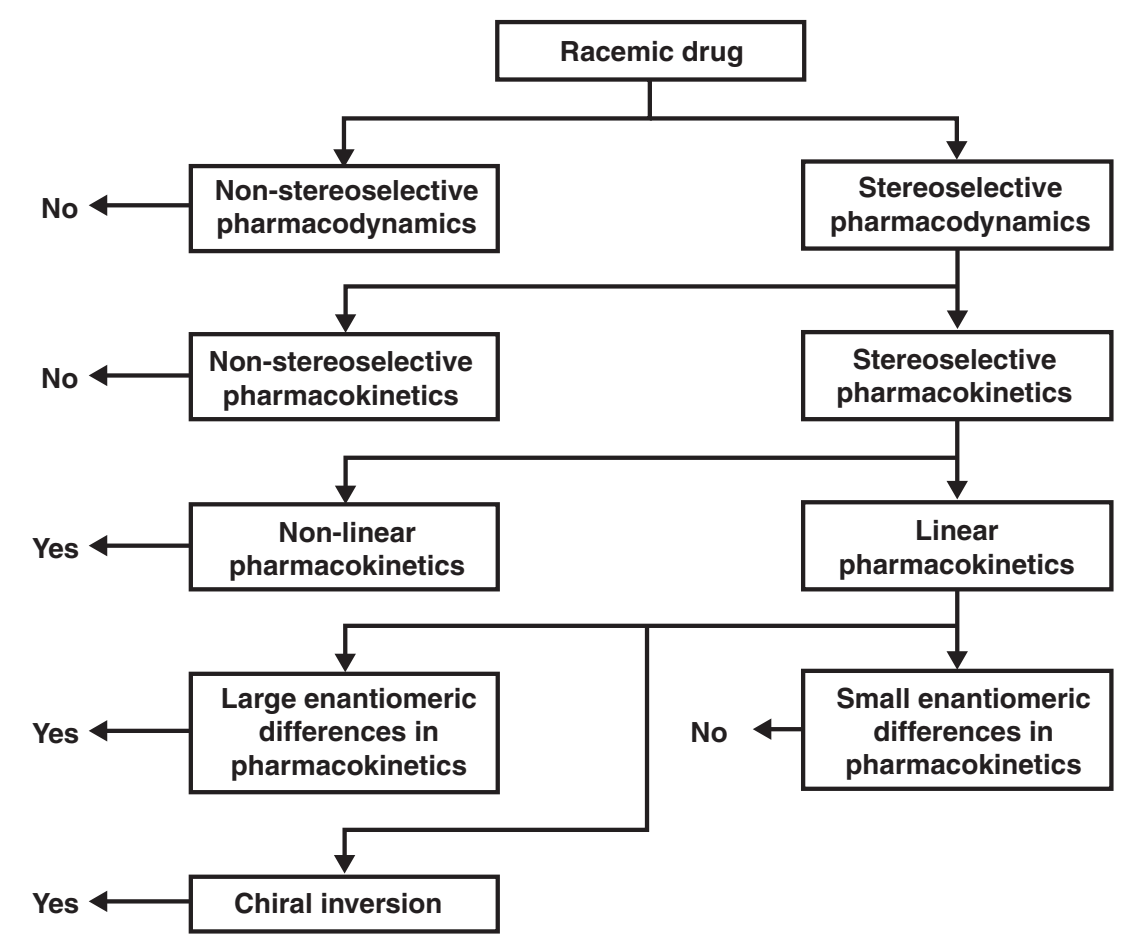

be affected by the specificity of the analytical method. Based on this evidence, we suggest that a decision tree be considered when designing bioequivalence studies of chiral drugs. It should be noted that in our computer simulations, we have attempted to identify situations for which the possibility of erroneous bioequivalence decision based on the total drug (as opposed to individual enantiomers) exists. Therefore, only error-free data for the reference and test formulations were generated and actual kinetic values (as opposed to confidence intervals used by regulatory agencies in the USA and Canada) were compared. However, in reality, a bioequivalence decision is influenced by both the actual magnitude of difference between the two formulations and the inter-and intra-individual variability in the kinetic parameters of the drug.

Concentrating on the important cases identified here, future studies should examine the effects of the degree of intra- and inter-individual variability and assay error on the bioequivalence decision of chiral drugs. The main question that needs to be answered is whether achiral assays are capable of demonstrating a lack of bioequivalence when one or more enantiomers of a chiral drug marginally lack bioequivalence. Nevertheless, more in-depth experimental and simulation studies are needed to test and refine the preliminary guidelines proposed in this and other communications.

In addition to considering stereoselective processes, stereospecific analytical methods also possess greater statistical power than do methods based on the total drug. This is because the differences in only one enantiomer become smaller when calculated as a percentage of the total drug than when expressed in terms of single enantiomer concentration.

It will be noted that the above algorithms refer to oral dosing of drugs and depend on the extent of first-pass metabolism. In veterinary therapeutics, on the other hand, many products are formulated for parenteral (e.g. intravenous, intramuscular, subcutaneous) use and others are formulated for systemic action after topical application (e.g. pour-on products) or as implants. For the majority of such products, there will be no first-pass effect (such as may occur within the gastrointestinal tract, or in enterocytes, lung or liver for orally administered drugs). Moreover, chemical degradation, at differing rates for an enantiomeric pair, at the parenteral or topical administration site is possible but unlikely. Therefore, if the algorithms are accepted, almost all nonoral bioequivalence studies could be conducted by measuring 'total drug' for such formulations. However, in a recent review, Heard and Brain (2010) pointed out that the stratum corneum, the rate limiting barrier to percutaneous administration, comprises several components (mainly keratin and ceramides), which are potential sources of chiral discrimination. Different local diffusion rates are therefore possible, depending on solute stereochemistry.

Nerurkar et al. (1992) and Srinivas (2004) review several articles, in which stereoselective pharmacogenetics in the disposition of drug racemates has been explored. A concern with use of a nonstereoselective assay is the possibility of failing to detect true disposition differences of the drug in the patient population predisposed to genetic polymorphism. For example, it is known that age-related changes in CYP2C19 activities can occur. This led Srinivas (2004) to conclude not only that stereoselective analysis would be preferable for drugs displaying stereoselective pharmacogenetics but also that the selection of subjects/animals from the population should be considered. He suggested that it may not be sufficient, even if possible, to select subjects of a single phenotype. Against this should be mentioned the assumption underlying bioequivalence determination in animals, that a species-drug interaction is formally hypothe- 
sized, whilst within-species differences are not, unless differences are relatively extreme, for example pre-ruminant calves vs. adult lactating cattle.

\section{Linear vs. nonlinear pharmacokinetics}

Srinivas (2004) proposed that the starting point for deciding on use of a stereoselective or nonstereoselective assay in bioequivalence studies is detailed knowledge of the pharmacological profile of the enantiomers. He indicated that this involves (i) quantitative data on their potency for the pharmacological actions underlying therapeutic use (and hence potency ratios) and side effects and (ii) based on their pharmacokinetic profile, estimating the contribution of each enantiomeric plasma/blood AUC to overall effect. Srinivas (2004) further emphasized the importance of knowledge of (i) any stereoselective presystemic metabolism of the components of the racemate and the saturability of the processes involved; (ii) other active and passive processes, including saturability, of elimination processes; (iii) the extent of plasma protein binding and effect of changes in binding on free-fraction equilibrium on enantiomer distribution and clearance; and (iv) the formation of active chiral metabolites from chiral or nonchiral parent drugs. This information may be useful in understanding the pharmacokinetic behaviour of drug racemates at different dosage rates and varied input rates from, for example, tablets, capsules and solutions.

Nonlinear pharmacokinetics occurs when dose increase or repeat dosing leads to a disproportionate increase in AUC (saturable clearance) or conversely to a lack of proportional increase of AUC in the case of saturable absorption. In humans, this has been documented for several drugs, including propranolol, phenytoin, theophylline and verapamil. Like Karim (1996) and Midha et al. (1998), Mehvar and Jamali (1997) reviewed case studies, supported by simulations, as a basis for comparing stereoselective vs. nonstereoselective methods for assessing bioequivalence. They concluded that individual enantiomers should be quantified for drug racemates that exhibit (i) nonlinear pharmacokinetics; or (ii) pronounced enantioselective differences in pharmacokinetic profiles, despite displaying linear pharmacokinetics; and (iii) in vivo chiral inversion. On the other hand, they recommend the use of nonstereoselective methods for drug racemates with linear pharmacokinetic profiles and when there is minimal stereoselectivity in pharmacokinetic parameters.

\section{Conclusions on active constituents from case histories}

The scientific literature contains many more examples of stereoisomerism in relation to bioequivalence in humans than in nonhuman animals. Some human studies are considered here, because the principles are independent of species, so that conclusions reached will be relevant to veterinary medicine, provided the human studies are adequately designed. However, there are some specific veterinary issues, arising for example from novel dosage forms and formulation-determined modified drug-release characteristics. As Midha et al. (1998) point out, appropriate design and statistical analysis are not always assured. They cite a bioequivalence publication on flurbiprofen in which paired t-tests led to the conclusion that stereoselective analysis was judged to be most appropriate. As Midha et al. (1998) emphasize, however, the paired t-test is based on a null hypothesis of no difference between two products, whereas the required two one-sided test procedure depends on a null hypothesis of inequivalence between the formulations, which must be rejected for the alternative hypothesis of bioequivalence to be accepted.

\section{Flurbiprofen.}

Flurbiprofen is a NSAID of the 2-arylpropionate subclass, used in humans as the racemic mixture. It exists in two $\mathrm{S}(+)$ and (R-)enantiomeric forms. In vivo, there is unilateral inversion of $\mathrm{S}(+)$ to $\mathrm{R}(-)$-flurbiprofen. However, as there is negligible nonstereoselective first-pass metabolism (enantiomeric difference in AUC is $<20 \%$ ), it is a category 1 drug (Fig. 3), so that a nonstereoselective analytical method should give reliable data, according to Midha et al. (1998) concluded that this was so, for the orally administered racemate, as all confidence intervals for nonstereoselective and stereoselective methods fell within the required range of $80-125 \%$ (Table 2). However, this conclusion might be questioned on two grounds. First, no specific consideration was given to the fact that most and probably all of the efficacy depends on the $S(+)$-enantiomer. Secondly, it is indeed the case that the required confidence intervals were met by both stereoselective and nonstereoselective assays, but they were not identical. For AUC, confidence intervals were 99.8-106.2 (total drug), 101.3-115.4 (S-enantiomer) and 99.0-116.4 (Renantiomer). The upper confidence intervals were thus greater for the separate enantiomers. What conclusions would have been arrived if all confidence intervals had been somewhat wider and if the upper confidence interval had marginally exceeded $125 \%$, for one or both enantiomers but not for total drug?

\section{Propafenone.}

Propafenone is a chiral anti-dysrhythmic drug, licensed for human use as the racemate. It undergoes extensive first-pass metabolism and exhibits oxidative polymorphism. There is no enantioselective first-pass metabolism (category 1 drug). In a bioequivalence study, confidence intervals for total drug and

Table 2. $90 \%$ Confidence intervals calculated for the data on two formulations of flurbiprofen (Midha et al., 1998)*

\begin{tabular}{lc}
\hline Assay and variables & $90 \%$ Confidence intervals \\
\hline Nonsteroselective & $99.8-106.2$ \\
$\ln$ AUC & $89.3-105.4$ \\
$\ln C_{\max }$ & \\
$\quad$ S-enantiomer & $101.3-115.4$ \\
$\ln$ AUC & $89.1-106.4$ \\
$\ln C_{\max }$ & \\
R-enantiomer & $99.0-116.4$ \\
$\ln$ AUC & $90.1-108.2$ \\
$\ln C_{\max }$ & \\
\hline
\end{tabular}

AUC, area under curve. 
Table 3. $90 \%$ Confidence intervals in a bioequivalence study on two formulations of propafenone conducted by Koytchev et al. (1995)

\begin{tabular}{lc}
\hline Assay and variables & $90 \%$ Confidence intervals \\
\hline Nonsteroselective* & $84-107$ \\
$\ln$ AUC & \\
S-propafenone & $83-110$ \\
$\ln$ AUC & $82-117$ \\
$\ln C_{\max }$ & \\
R-propafenone & $85-107$ \\
$\ln$ AUC & $81-112$ \\
$\ln C_{\max }$ & \\
5 -HO-propafenone & \\
$\ln$ AUC & $84-105$ \\
$\ln C_{\max }$ & $87-109$ \\
\hline
\end{tabular}

AUC, area under curve. *Calculated by Midha et al. (1998) by summing the stereoselective data. ${ }^{\dagger}$ Nonstereoselective assay used.

each enantiomer were within $80-125 \%$ limits, but (as with flurbiprofen, vide supra) they were not identical and lower limits for $C_{\max }$ approached the lower $80 \%$ level (Table 3). Nevertheless, Midha et al. (1998) concluded that a nonstereoselective assay would be acceptable.

\section{Nadolol.}

Like endogenous catecholamines (adrenaline, noradrenaline), which act on adrenoceptors of various types and subtypes, many antagonists for $\beta$-adrenoceptors contain a single chiral centre. The eutomer is the L-antipode, and no significant $\beta$-receptor blocking activity resides in the D-enantiomer. Nadolol possesses two chiral centres. Therefore, there are four optical isomers, differing in potencies for inhibition at $\beta$-adrenoceptors. It is a category 1 drug as there is no significant first-pass effect. Bioavailability is relatively low (of the order of 35\%). Midha et al. (1998) suggested that stereoselective transport mechanisms may be involved in the absorption of nadolol from the gastrointestinal tract, favouring the use of a stereoselective analytical method.

Data collected using both non- and stereoselective assays for two oral formulations were evaluated for bioequivalence in single-dose (Table 4) and multiple-dose (steady state) studies (Srinivas et al., 1996). 90\% Confidence intervals were within the 80-125\% range for $C_{\max }$ and AUC for both single- and multipledose studies for total drug. For the stereoisomers, on the other hand, bioequivalence was achieved for $C_{\max }$ for three of four isomers and for AUC for only two of four isomers.

This example illustrates (on an initial consideration) the likely requirement for stereoselective methodology, even for a category 1 compound. However, Midha et al. (1998) noted that the within-subject variability of concentrations of stereoisomers was greater than that of total drug. This raises the possibility that some or all of the failure of stereoisomeric assessments to conclude bioequivalence was attributed to low statistical power. This could arise if high assay variability increased the variability of the residual square mean ANOvA, leading to wider $90 \%$ confidence intervals and resulting in failure to meet the 80$125 \%$ range. In fact, there was greater variability of the ANOvA-
Table 4. $90 \%$ Confidence intervals in a bioequivalence study on two formulations of nadolol after single oral doses (Srinivas et al., 1996)

\begin{tabular}{lc}
\hline Assay and variables & $90 \%$ Confidence intervals \\
\hline Total nadolol* & \\
$\ln$ AUC & $93-116$ \\
$\ln C_{\max }$ & $84-117$ \\
SQ-12148 & \\
$\ln$ AUC & $86-116$ \\
$\ln C_{\max }$ & $72-106^{\ddagger}$ \\
SQ-12149 & \\
$\ln \mathrm{AUC}$ & $79-137^{\ddagger}$ \\
$\ln C_{\max }$ & $81-119$ \\
SQ-12150 & \\
$\ln \mathrm{AUC}$ & $82-119$ \\
$\operatorname{lkn} C_{\max }$ & $89-122$ \\
$\mathrm{SQ}-12151^{\dagger}$ & \\
$\ln \mathrm{AUC}$ & $71-102^{\ddagger}$ \\
$\ln C_{\max }$ & $82-117$ \\
\hline
\end{tabular}

AUC, area under curve. *Nonstereoselective method. ${ }^{\dagger}$ Stereoselective method. ${ }^{*}$ The figures in bold are not bioequivalent.

CV\% associated with the stereospecific assays than with the total drug method; for the individual enantiomers, after single doses, ANOVA-CVs were $87 \%, 52 \%, 51 \%$ and $41 \%$, whilst that for the nonstereoselective method was $22 \%$. This added variability for individual stereoisomers was not attributable to the pharmacokinetic component of the ANOVA-CV averaged across the enantiomers or to formulation factors, which did not differ for the two treatments. It was concluded that the added variability was because of greater variability in the stereoselective assay method, and failure of confidence intervals to fit within the range 80$125 \%$ was due primarily to low statistical power.

\section{Nortriptyline.}

Nortryptyline possesses antidepressant CNS activity, but it is also a pro-drug, forming active metabolites. It is a nonchiral molecule, which undergoes metabolic oxidation; the 10-hydroxymetabolite exhibits both optical and geometric isomerism. Both geometric isomers of the 10-hydroxy metabolite possess biological activity, but E-10-hydroxynortriptyline possesses less pronounced side effects than nortriptyline. E-10-hydroxynortriptyline is the major form present in urine, the Z-10 isomer contributing $<20 \%$ of the total hydroxylation capacity of human hepatic microsomes.

In a bioequivalence study using capsule formulations of nortriptyline, ( \pm )-E-10- and ( \pm )-Z-10-hydroxynortriptyline were quantified in an assay that separated the optical isomers as well as nortriptyline (Table 5; Midha et al., 1995). For parent compound, $90 \%$ confidence intervals were narrow and well within the required $80-125 \%$ bounds, and on this ground, analysis of parent compound alone would be justified. The analysis of total 10-hydroxynortriptyline also confirmed that bioequivalent criteria were met, as also did the confidence intervals for $( \pm)$-E-10-hydroxynortriptyline. However, for the ( \pm )-Z-10-hydroxy isomer, the lower confidence interval for AUC was 79.4 . Review of the analytical methods revealed that only 
Table 5. 90\% Confidence intervals in a bioequivalence study on two formulations of nortriptyline (Midha et al., 1995)

\begin{tabular}{|c|c|}
\hline Assay and variables & $95 \%$ confidence intervals \\
\hline \multicolumn{2}{|l|}{ Nortriptyline } \\
\hline In $\mathrm{AUC}_{\text {last }}$ & $95.8-105.6$ \\
\hline $\ln C_{\max }$ & $95.2-105.9$ \\
\hline \multicolumn{2}{|c|}{ Total 10-hydroxynortriptyline } \\
\hline ln $\mathrm{AUC}_{\text {last }}$ & $92.2-103.4$ \\
\hline $\ln C_{\max }$ & $92.8-112.1$ \\
\hline \multicolumn{2}{|c|}{ (E)-10-hydroxynortriptyline } \\
\hline In $\mathrm{AUC}_{\text {last }}$ & $91.8-103.2$ \\
\hline $\ln C_{\max }$ & $91.6-111.4$ \\
\hline \multicolumn{2}{|c|}{ (Z)-10-hydroxynortriptyline } \\
\hline $\ln \mathrm{AUC}_{\text {last }}{ }^{*}$ & $79.4-112.3^{\dagger}$ \\
\hline $\ln C_{\max }$ & $95.9-110.8$ \\
\hline
\end{tabular}

AUC, area under curve. *Highly variable (ANOVA-CV 33.3\%). ${ }^{\dagger}$ The figures in bold are not bioequivalent.

( \pm )-Z-10-hydroxynortriptyline was highly variable (ANOvA$\mathrm{CV}=33.3 \%)$. In consequence, the failure to declare bioequivalence for this metabolite alone was judged to be the result of low statistical power and was not an indication that the stereoselective analytical method was more discriminatory.

\section{Hydroxychloroquine.}

Hydroxychloroquine contains a single chiral centre and is used commercially as an antirheumatic drug as the racemate. Because, in humans, each enantiomer accumulates in erythrocytes, whole blood elimination half-lives are long; 25.5 days for the R-enantiomer and 16.7 days for the S-enantiomer (Brocks et al., 1994). Based on the algorithm of Fig. 3, hydroxychloroquine could be classified as a category I (nonsignificant first-pass metabolic effect) or category IIb (specific isomer ratio not important on the basis of stereoselective renal clearance) drug. Subject to acceptance of the algorithm, a nonstereoselective analytical method is justified.

Using a parallel study design in human males, test and pioneer formulations of the racemate were compared, with AUC for whole blood determined over an 11-week sampling period. Concentrations were monitored by both nonselective and stereoselective methods. Ninety percentage confidence intervals were based on between- rather than within-subject variances, because of the parallel study design (Midha et al., 1996). The same bioequivalence decisions were reached for both analytical methods (Table 6). However, confidence intervals were dissimilar for unknown reasons; in particular, for AUC, the upper confidence intervals were higher for both enantiomers than for total drug measured nonstereoselectively.

This example is of interest because of the long terminal halflives. Bioequivalence concerns demonstration of similarity of rate and extent of absorption, and for a drug of this kind, the greater proportion of AUC is determined by its slow clearance. Therefore, it was of interest to note that for all analytes, chiral and nonchiral, the $\mathrm{AUC}_{0-4}$ days as well as $\mathrm{AUC}_{0-\infty}$ provided $90 \%$ confidence intervals within the range $80-125 \%$. This case
Table 6. 90\% Confidence intervals calculated for data on two formulations of hydroxychloroquine (Midha et al., 1996)

\begin{tabular}{lc}
\hline Assay and variables & $95 \%$ Confidence interval \\
\hline Nonstereoselective & \\
$\quad \ln$ AUC & $85.36-105.8$ \\
$\ln C_{\max }$ & $86.35-109.2$ \\
(R)-hydroxychloroquine & \\
ln AUC & $84.53-115.4$ \\
$\ln C_{\max }$ & $86.02-112.2$ \\
(S)-hydroxychloroquine & \\
$\ln$ AUC & $85.04-117.7$ \\
$\ln C_{\max }$ & $86.07-111.9$ \\
\hline
\end{tabular}

AUC, area under curve.

illustrates, for bioequivalence purposes, the use of a shorter sampling time and the derivation of partial areas for AUC (Midha et al., 1996). More recently, Najib et al. (2009) investigated the use of truncated AUC on bioequivalence outcome for nine model drugs.

This example raises other issues of principle. Achiral assays measure both enantiomers, but at and beyond some point, the assay will only measure one of them, if they have different clearance rates. Therefore, the same compound would not be measured throughout the sampling profile. Likewise, there will be an issue, if the two enantiomers have different $T_{\max }$ values. These considerations are additional issues of complexity for long half-life drugs, either because of the active pharmaceutical ingredient (API) or of the formulation (Gehring and Martinez, personal communication). Finally, there is the question of the approach to be taken if the starting API is not a racemate but some other proportion of the enantiomers. In this circumstance, stereospecific methods would be preferred.

\section{Carprofen.}

The pharmacokinetic profiles of carprofen enantiomers in dogs were investigated by Priymenko et al.(1998). Rac-carprofen was administered intravenously to dogs with surgically implanted bile duct catheters. Percentage recoveries of the administered dose recovered from bile were $74(\mathrm{R}(-)$-enantiomer) and 92 $(\mathrm{S}(+)$-enantiomer), as the respective glucuronide conjugates. It was further shown that carprofen was subjected to enantioselective enterohepatic recycling, with only the $\mathrm{S}(+)$-enantiomer being recycled.

The same group investigated the influence of feeding and analytical method on the bioequivalence of carprofen (Priymenko et al., 2004). They used a single racemic product to determine the effect of feeding and analytical method (enantioselective vs. nonenantioselective) on statistical power of the studies. One trial used a standard feeding protocol and a second used a special feeding protocol selected to ensure constant biliary flow into the duodenum. Using a nonenantioselective technique, $90 \%$ confidence intervals provided conclusions of bioequivalence in $100 \%$ of the cases for both AUC and $C_{\max }$ with the special feeding protocol, but only $50 \%$ for AUC and $13 \%$ for $C_{\max }$ with the standard feeding protocol, indicating that a feeding pattern 
that reduces plasma carprofen concentration rebound for an enterohepatically recycled drug increased the power of a bioequivalence trial. Irrespective of the feeding protocol, enantioselective analysis decreased the power of the trials for AUC but increased the power for $C_{\max }$. Thus, for an enterohepatically recycled drug, feeding pattern influenced the power of the bioequivalence trial, and the analytical technique that provided the greatest power depended on the assessed bioequivalence parameter and the feeding pattern.

\section{Influence of pulsatile input on selection of analytical method}

There are several ways in which formulation differences (product classes, e.g. tablets vs. capsules, excipients and solvents) can impact on the choice of the most appropriate analytical method for bioequivalence studies. Mehvar (1994) undertook computer simulations to test the effect of pulsatile oral input on the stereoselectivity of AUC in blood on enantiomers of racemic drugs. He found that enantiomeric AUC ratios were dependent on all determinants of input rate. He concluded that bioequivalence studies based on total drug measurement may result in erroneous conclusions for the individual enantiomers.

In veterinary medicine, controlled-release products have been developed for extended absorption from parenteral (subcutaneous or intramuscular sites) routes as well as the use of controlled-release devices placed in the rumen. For such products, stereoselective absorption differences between generic and pioneer products cannot be excluded. Controlled-release devices are in common use, for example for delivery of anthelmintics into the rumen of cattle and sheep in a regular or pulsatile manner (Brayden et al., 2010). If, in such products, drug racemates are used, a stereoselective method may be required in bioequivalence evaluation to detect subtle but important stereoselective differences in presystemic absorption.

\section{Impact of excipients and solvents on bioequivalence}

Several excipients used in pharmaceutical formulations are chiral, raising the possibility of interaction with chiral drugs formulated as racemates. Chiral excipients include sucrose, tartaric acid, methylcellulose, hydroxypropylmethyl cellulose and hydroxypropyl- $\beta$-cyclodextrin (HPCD). Janjikhel and Adeyeye (1999) investigated the dissolution of ibuprofen enantiomers from coprecipitates and suspensions containing chiral excipients. Any such influence would have implications for bioequivalence determination. In the case of ibuprofen, it was found that dissolution profiles of coprecipitates were higher than those of pure ibuprofen. However, the presence of chiral excipients (HPCD and tartaric acid) did not lead to stereoselective release of ibuprofen enantiomers from either coprecipitates or suspensions.

Nerurkar et al. (2005) investigated the saturation solubilities of rac-ibuprofen and $\mathrm{S}(+)$-ibuprofen in the presence of cosolvents and cyclodextrins as inclusion-complexing agents. Ibuprofen is virtually insoluble in water. In vitro studies were conducted using aqueous solutions, in which organic co-solvents or complexing agents (three cyclodextrins) were used to enhance the solubility of rac-ibuprofen and its S-enantiomer. It was postulated that the use of organic solvents, such as propylene glycol and polyethylene glycol or the cyclodextrins, might minimize gastrointestinal tract irritation of ibuprofen by increasing dissolution rate and thereby increasing absorption rate. Moreover, as $\mathrm{S}(+)$-ibuprofen is the eutomer for both therapeutic and side effects, it is of interest to determine any differences in solubilities between the racemate and the S-antipode, such differences potentially impacting on bioequivalence of chiral drug molecules. Propylene glycol increased the solubilities of racand S(+)-ibuprofen by 193 - and 400 -fold at $25^{\circ} \mathrm{C}$ respectively. Corresponding increases in solubility achieved by polyethylene glycol 300 were 700- and 1500-fold. These increases were achieved with the highest concentrations $(80 \% \mathrm{v} / \mathrm{v})$ of cosolvents used.

Compared with rac-ibuprofen, the $\mathrm{S}(+)$-enantiomer formed more stable complexes with HPCD and $\beta$-cyclodextrinsulphobutyl ether sodium salt (CDSB). The increased solubility in the presence of $25 \% \mathrm{w} / \mathrm{v}$ HPCD was 175 - and 242-fold for rac- and $\mathrm{S}(+)$-ibuprofen, respectively. The corresponding increases in solubility for CDSB were 113- and 175-fold (Nerurkar et al., 2005).

Suedee et al. (2002) considered another potential influence of excipients on bioequivalence of stereoisomers. They investigated the stereoselective release behaviours of low-swelling molecularly imprinted polymer (MIP) bead matrices in pressed-coat tablets. The model drug used was the $\beta$-adrenoceptor antagonist propranolol. It was concluded that the enantioselective-controlled delivery mechanism of MIPs depends on the relative affinity of the enantiomer for the template sites, as well as the nature of the polymer, such as hydrophobicity and swellability.

\section{Fixed combination products}

Combination products containing two active constituents in fixed dose proportions are used in both human and veterinary medicine. One or both actives might contain a chiral centre. In this circumstance, one drug might influence the absorption of a second drug in a stereoselective manner, although the authors are not aware of such a report.

\section{Drug interactions}

As with fixed combination products, it may be necessary to consider interactions between drugs of different classes, which are commonly co-administered. Ketoprofen is an extensively used analgesic agent in human and veterinary medicine. Because an important side effect is upper gastrointestinal tract irritation, it is common to advise therapeutic use in combination with proton pump inhibitors such as omeprazole, to minimize such side effects. Qureshi et al. (1994) therefore studied the influence of co-administration of omeprazole (single dose) on the pharmacokinetics of two enteric-coated ketoprofen tablets to establish any influence on absorption of one or both of the ketoprofen products, which had previously been shown to be bioequivalent. There was a trend towards higher AUC values for 
both S+ and R-ketoprofen, in the presence of omeprazole, but this small effect was similar for both ketoprofen products.

\section{CONCLUSIONS AND CURRENT REGULATORY VIEWS}

In lieu of the algorithm described in Figs 3, and 4, it may seem to be an attractive alternative to use stereospecific methods whenever possible, and especially in circumstances where the biological activity (pharmacological or toxicological) is known to reside primarily or solely in a single enantiomer. This way, it can be argued that therapeutic similarity between test and reference products will be best assured (Hooper et al., 1992; Hooper, 1993). However, to do so greatly increases the risk of failure to demonstrate product bioequivalence due simply to chance. Therefore, the conditions under which the use of stereospecific methods should be required need to be carefully defined. Unfortunately, there remain many unanswered questions that constrain our ability to delineate such a list of recommendations.

Srinivas (2004) emphasizes that controversy and debate still surround the question whether to use or not to use stereoselective methods in bioequivalence studies now that they are so widely available. Proponents of the use of stereoselective analysis argue that, if pharmacological and/or toxicological activity of the racemate is due primarily or solely to one enantiomer (which is commonly the case), it is rational to analyse the eutomer in preference to total drug. Opponents, on the other hand, argue that stereoselective assays add to development costs and that in most circumstances measurement of the individual antipodes will not alter the decision on bioequivalence outcome, because the racemates in both test and reference products have, by definition, the same fixed ratio of the two enantiomers. Between these extremes is the argument for developing guidelines, based on the algorithms (Figs 3 and 4) together with consideration of individual case histories.

One point to note is that, for reasons expanded upon in our background material, achiral assays should not be used to support regulatory decisions to support a change in the route of administration (e.g. parenteral to oral or vice versa). Similarly, achiral assays should be discouraged when relative bioavailability concepts are used to support interspecies extrapolations (primarily associated with minor species drug approvals).

Currently, the European (EMA, 2010) and the USA (FDA) regulatory authorities offer somewhat different guidance with regard to the choice of analytical methods for use in bioequivalence studies, when the products contain chiral compounds. EMA has presented for discussion new guidelines on bioequivalence. The draft (2010) document states,

the use of achiral bioanalytical methods is generally acceptable. However, the individual enantiomers should be measured when all the following conditions are met:

a) the enantiomers exhibit different pharmacokinetics

b) the enantiomers exhibit pronounced differences in pharmacodynamics

c) the exposure (AUC) ratio of enantiomers is modified by a difference in the rate of absorption.
If one enantiomer is pharmacologically active and the other is inactive or has a low contribution to activity, it is sufficient to demonstrate bioequivalence for the active enantiomer. Further, the use of achiral bioanalytical methods is possible when both products contain the same single enantiomer and there is not interconversion in vivo.

In the USA, FDA (2006) recommends measurement of the racemate using an achiral assay as the basic position. Measurement of individual enantiomers in bioequivalence studies is recommended only when all the following conditions are met: (i) the enantiomers exhibit different pharmacodynamic characteristics; (ii) the enantiomers exhibit different pharmacokinetic characteristics; (iii) primary efficacy/safety resides with the minor enantiomer; and (iv) nonlinear absorption is present (as expressed by a change in the enantiomer concentration ratio with change in the input rate of the drug) for at least one of the enantiomers. In such cases, bioequivalence criteria should be applied to the separate enantiomers.

Based on our assessments as described in this manuscript, we strongly encourage regulators to more carefully explore these recommendations, particularly from the perspective of the novel dosage forms and modified drug-release characteristics associated with many veterinary formulations.

\section{CONFLICTS OF INTEREST}

R.P.H. is employed by Elanco Animal Health; the remaining authors declare no conflicts of interest.

\section{REFERENCES}

Ariens, E.J. (1986) Chirality in bioactive agents and its pitfalls. Trends in Pharmaceutical Sciences, 7, 200-205.

Barrett, J.S., Batra, V., Chow, A., Cook, J., Gould, A.L., Heller, A.H., Lo, M.W., Patterson, S.D., Smith, B.P., Stritar, J.A., Vega, J.M. \& Zariffa, N. (2000) PhRMA perspective on population and individual bioequivalence. Journal of Clinical Pharmacology, 40, 561-572.

Berry, B.W. \& Jamali, F. (1991) Presystemic and systemic chiral inversion of $\mathrm{R}(-)$ fenoprofen in the rat. Journal of Pharmacology El Experimental Therapeutics, 25B, 695-701.

Brayden, D.J., Oudet, E.M. \& Baird, A.W. (2010) Drug delivery systems in domestic animal species. In Comparative and Veterinary Pharmacology. Eds Cunningham, F.M., Elliott, J. \& Lees, P., pp. 79-112. SpringerVerlag, Berlin, Germany.

Brocks, D.R. (2006) Drug disposition in three dimensions: an update on stereoselectivity in pharmacokinetics. Biopharmaceutics \& Drug Disposition, 27, 387-406.

Brocks, D.R., Skeith, K.J., Johnston, C., Emamibafrani, J., Davis, P., Russell, A.S. \& Jamali, F. (1994) Hematologic disposition of hydroxychloroquine enantiomers. Journal of Clinical Pharmacology, 34, 1088-1097.

Cahn, R.S., Ingold, C.K. \& Prelog, V. (1956) The specification of asymmetric configuration in organic chemistry. Experientia, 12, 81124.

Caldwell, J., Hutt, A.J. \& Fournel-Gigleux, S. (1988) The metabolic chiral inversion and dispositional enantioselectivity of the 2-arylpropionic acids and their biological consequences. Biochemical Pharmacology, 37, 105-114. 
Campbell, D.B. (1990) Stereoselectivity in clinical pharmacokinetics and drug development. European Journal of Drug Metabolism and Pharmacokinetics, 15, 109-125.

Day, R.O., Williams, K.M., Graham, G.G., Lee, E.J., Knihinicki, R.D. \& Champion, G..D. (1988) Stereoselective disposition of ibuprofen enantiomers in synovial fluid. Clinical Pharmacology and Therapeutics, 43, 480-487.

Delatour, P., Benoit, E., Caude, M. \& Tambutu, A. (1990a) Species differences in the generation of the chiral sulphoxide metabolite of albendazole in sheep and rats. Chirality, 2, 156-160.

Delatour, P., Benoit, E., Garnier, F. \& Besse, S. (1990b) Chirality of the sulphoxide metabolites of fenbendazole and albendazole in sheep. Journal of Veterinary Pharmacology and Therapeutics, 13, 361-366.

Delatour, P., Benoit, E., Besse, S. \& Boukraa, A. (1991a) Comparative enantioselectivity in the sulphoxidation of albendazole in man, dogs and rats. Xenobiotica, 21, 217-221.

Delatour, P., Garnier, E., Benoit, E. \& Caude, I. (1991b) Chiral behaviour of the metabolite albendazole sulphoxide in sheep, goats and cattle. Research in Veterinary Science, 50, 134-138.

Delatour, P., Benoit, E., Besse, S. \& Soraci, A. (1994) Asymetrie moléculaire et pharmacologie comparee. Revue Medécine Vétérinarie, 145. $551-561$.

EMA (2010) Draft Guideline on the conduct of bioequivalence studies for veterinary medicinal products. EMEA/CVMP/016/00-Rev. 2-Consultation.

Evans, A.M. (1992) Enantioselective pharmacodynamics and pharmacokinetics of chiral non-steroidal anti-inflammatory drugs. European Journal of Clinical Pharmacology, 42, 237-256.

Gould, A.L. (2000) A practical approach for evaluating population and individual bioequivalence. Statistics in Medicine, 19, 2721-2740.

Heard, C.M. \& Brain, K.R. (2010) Does solute stereochemistry influence percutaneous penetration? Chirality, 7, 305-309.

Hooper, W.D. (1993) Are enantioselective assay methods necessary for comparative bioavailability studies on racemic drugs? Proceedings of Western Pharmacology Society, 36, 167-171.

Hooper, W.D., Dickinson, R.G. \& Gal, J. (1992) Enantioselective versus non-enantioselective assays in comparative bioavailability studies with racemic drugs. Biopharmaceutics \& Drug Disposition, 13, 383-387.

Ilett, K.F., Tee, L.G., Reeves, P.T. \& Minchin, R.F. (1990) Metabolism of drugs and other xenobiotics in the gut lumen and wall. Pharmacology and Therapeutics, 46, 67-93.

Jamali, F. (1992) Stereochemistry and bioequivalence. Journal of Clinical Pharmacology, 32, 930-934.

Jamali, F., Collins, D. S., Berry, B. W., Molder, S., Cheung, R., McColl, K. \& Cheung, H (1991) ???? Biopharmacology Drug Disposition, 12, 435445.

Janjikhel, R.K. \& Adeyeye, C.M. (1999) Dissolution of ibuprofen enantiomers from coprecipitates and suspensions containing chiral excipients. Pharmaceutical Development \& Technology, 4, 9-17.

Karim, A. (1996) Enantioselective assays in comparative bioavailability studies of racemic drug formulations: nice to know or need to know. Journal of Clinical Pharmacology, 36, 490-499.

King, J.N., Mauron, C., LeGoff, C. \& Hauffe, S. (1994) Bidirectional chiral inversion of the enantiomers of the non-steroidal anti-inflammatory drug oxindanac in dogs. Chirality, 6, 460-466.

Koytchev, R., Alken, R.G., Mayer, O., Bohm, R., Ellrich, A. \& Waldnerkolblin, R.G. (1995) Bioequivalence studies of two oral propafenone preparations. Arzneimittel Forschung, 45, 542-545.

Landoni, M.F., Soraci, A.L., Delatour, A. \& Lees, P. (1997) Enantioselective behaviour of drugs used in domestic animals: a review. Journal of Veterinary Pharmacology and Therapeutics, 20, 1-16.

Lees, P., Landoni, M.F., Giraudel, J. \& Toutain, P.L. (2004) Pharmacodynamics and pharmacokinetics of nonsteroidal anti-inflammatory drugs in species of veterinary interest. Journal of Veterinary Pharmacology \& Therapeutics, 27, 479-490.

Lötsch, J., Geisslinger, G., Mohammadian, P., Brune, K. \& Kobal, G. (1995) Effects of flurbiprofen enantiomers on pain-related chemosomatosensory evoked potentials in human subjects. British Journal of Clinical Pharmacology, 40, 339-346.

Marzo, A. (1993) How incoming guidelines on chiral drugs could impact on the international scenary of drug development. Bollettino Chimico Farmaceutico, 132, 267-271.

Mehvar, R. (1994) Input rate-dependent stereoselective pharmacokinetics: effect of pulsatile oral input. Chirality, 6, 185-195.

Mehvar, R. \& Jamali, F. (1997) Bioequivalence of chiral drugs: stereospecific versus non-stereospecific methods. Clinical Pharmacokinetics, 33, 122-141.

Midha, K.K., Hubbard, J.W., McKay, G., Rawson, M. \& Schwede, R. (1995) Stereoselectivity in bioequivalence studies of nortriptyline. European Journal of Pharmaceutical Sciences, 84, 1265-1266.

Midha, K.K., Hubbard, J.W., Rawson, M.J. \& Schwede, R. (1996) The roles of stereochemistry and partial areas in a parallel design study to assess the bioequivalence of two formulations of hydroxychloroquine: a drug with a very long half-life. European Journal of Pharmaceutical Sciences, 4, 283-292.

Midha, K., McKay, G., Rawson, M.J. \& Hubbard, J.W. (1998) The impact of stereoisomerism in bioequivalence studies. Journal of Pharmaceutical Sciences, 87, 797-802.

Najib, N.M., Salem, I., Hasan, R. \& Idkaidek, N.M. (2009) Effect of truncated AUC method on drug bioequivalence in humans. Journal of Bioequivalence \& Bioavailability, 1, 112-114.

Nation, R.L. \& Sanson, L.N. (1994) Bioequivalence requirements for generic products. Pharmacology \& Therapeutics, 62, 41-55.

Nerurkar, S.G., Dighe, S.V. \& Williams, R.L. (1992) Bioequivalence of racemic drugs. Journal of Clinical Pharmacology, 32, 935-943.

Nerurkar, J., Beach, J.W., Park, M.O. \& Jun, H.W. (2005) Solubility of $( \pm)$-ibuprofen and $S(+)$-ibuprofen in the presence of cosolvents and cyclodextrins. Pharmaceutical Development \& Technology, 10, 413-421.

Nitchuk, W.M. (1992) Regulatory requirements for generic chiral drugs. Journal of Clinical Pharmacology, 32, 953-954.

Priymenko, N., Garnier, F., Ferre, J.P., Delatour, P. \& Toutain, P.L. (1998) Enantioselectivity of the enterohepatic recycling of carprofen in the dog. Drug Metabolism \& Disposition, 26, 170-176.

Priymenko, N., Koritz, G.D., Ferre, J.P., Laroute, M.F. \& Toutain, P.L. (2004) Influence of feeding and analytical method on the bioequivalence of a racemic drug undergoing enantioselective enterohepatic recycling. Journal of Pharmaceutical Sciences, 93, 590-600.

Qureshi, S., Caille, G., Lacasse, Y. \& McGilveray, I. (1994) Pharmacokinetics of two enteric-coated ketoprofen products in humans with or without coadministration of omeprazole and comparison with dissolution findings. Pharmaceutical Research, 11, 1669-1672.

Simonyi, M., Gal, J. \& Testa, B. (1989) Signs of the times: the need for a stereochemically informative generic name system. Trends in Pharmacological Sciences, 10, 349-354.

Soraci, A.L., Benoit, E., Jaussaud, P., Lees, P. \& Delatour, P. (1995) Enantioselective glucuronidation and subsequent biliary excretion of carprofen in horses. American Journal of Veterinary Research, 56, $358-$ 361.

Srinivas, N.R. (2004) Role of stereoselective assays in bioequivalence studies of racemic drugs: have we reached a consensus? The Journal of Clinical Pharmacology, 44, 115-119.

Srinivas, N.R., Barr, W.H., Shyu, W.C., Mohandoss, E., Chow, S., Staggers, J., Balan, G., Belas, F.J., Blair, I.A. \& Barbhaiya, R.H. (1996) Bioequivalence of two tablet formulations of nadolol using single and multiple dose data:assessment using stereospecific and nonstereospecific assays. Journal of Pharmaceutical Sciences, 85, 299-303. 
Suedee, R., Srichana, T., Chotivatesin, R. \& Martin, G.P. (2002) Stereoselective release behaviors of imprinted bead matrices. Drug Development and Industrial Pharmacy, 28, 545-554.

Tamai, I., Ling, H.Y., Timbul, S.M. \& Nishikido, T.A. (1988) Stereospecific absorption and degradation of cephalexin. Journal of Pharmacy and Pharmacology, 40, 320-324.

Tanaka, Y., Shimomura, Y., Hirota, T., Nozaki, A., Ebata, M., Takasaki, W., Sigehara, E., Hayashi, R. \& Caldwell, J. (1992) Formation of glycine conjugate and (-)- and (+)-enantiomers from (+)-(S)-2-phenylpropionic acid suggesting the formation of the CoA thioester intermediate in dogs. Chirality, 4, 342-348.

Testa, B., Carrup, P.A. \& Gal, J. (1993) The so called 'interconversion' of stereoisomeric drugs: an attempt at clarification. Chirality, 5, 105111.

Ticku, M.K., Rastogi, S.K. \& Thyagarajan, R. (1985) Separate sites of action of optical isomers of 1-methyl-5-phenyl-5-propylbarbituric acid with opposite pharmacological activities at the GABA receptor complex. European Journal of Pharmacology, 112, 1-9.
Toutain, P.L. \& Lees, P. (2004) Integration and modelling of pharmacokinetic and pharmacodynamic data to optimize dosage regimens in veterinary medicine. Journal of Veterinary Pharmacology \& Therapeutics, 27, 467-477.

Tucker, G.I. \& Lennard, M.S. (1990) Enantiomer specific pharmacokinetics. Pharmacology and Therapeutics, 45, 309-329.

Van't Hoff, J.H. (1898) The Arrangement of Atoms in Space. Longmans Green, London, UK.

Wang, Y., Cao, J., Wang, X. \& Zeng, S. (2010) Stereoselective transport and uptake of propranolol across human intestinal Caco-2 cell monolayers. Chirality, 22, 361-368.

Wechter, W.J. (1992) From controversy to resolution: bioequivalence of racemic drugs - A. bioequivalency and analytical aspects of stereochemistry. Journal of Clinical Pharmacology, 32, 915-916.

Williams, K., Day, R., Knihinicki, R. \& Duffield, A.P. (1986) The stereoselective uptake of ibuprofen enantiomers into adipose tissue. Biochemical Pharmacology, 35, 3403-3405. 